\title{
RESERVAS ECOLÓGICAS EN MÉXICO DESDE LA PERSPECTIVA DE LA MERCADOTECNIA SOSTENIBLE
}

\author{
ECOLOGICAL RESERVES IN MEXICO FROM THE PERSPECTIVE OF SUSTAINABLE \\ MARKETING
}

\author{
Ana Karen Huerta-Mendoza ${ }^{1}$ (iD); Laura Estela Fischer-de-la-Vega ${ }^{1}$ (D) . \\ 1. Facultad de Contaduría y Administración, Universidad Nacional Autónoma de México \\ karen.1392@hotmail.com y lfischer@fca.unam.mx
}

*Autor de Correspondencia: Ana Karen Huerta Mendoza, correo electrónico: karen.1392@hotmail.com.

\section{RESUMEN}

El crecimiento acelerado de la población, la necesidad imperante de abarcar más espacios para construir viviendas, plazas y edificios ha ido en aumento; sin embargo, los espacios verdes, como parques urbanos y reservas ecológicas, han sido reducidos o abandonados por las administraciones correspondientes para satisfacer las necesidades de la sociedad, con el objetivo de conocer la importancia del desarrollo y conservación de las Reservas Ecológicas o Áreas Naturales Protegidas buscando un aprovechamiento a través del ecoturismo, implementando estrategias de mercadotecnia sostenible se realizó un estudio cualitativo a través de la lectura de documentos de 182 páginas web de áreas naturales protegidas en México, analizando características, ecosistemas e instalaciones profundizando la revisión documental en las tres principales reservas ecológicas de Querétaro. Como principales hallazgos se encuentran la falta de una adecuada estrategia de sus recursos, el bajo porcentaje de presupuesto e inversión destinados al resguardo o mejora de ellas; haciendo que la mercadotecnia sostenible cobre una gran importancia en la administración de estas reservas.

Palabras clave: Reservas ecológicas; mercadotecnia sostenible; ecoturismo.

Cómo citar:

Huerta-Mendoza, Ana Karen; Fischer-de-la-Vega, Laura Estela. (2021). Reservas ecológicas en México desde la perspectiva de la mercadotecnia sostenible. Revista de Investigaciones Universidad del Quindio, 33(S1), 6-18. https://doi.org/10.33975/riuq.vol33nS1.479 


\begin{abstract}
The accelerated growth of the population, the prevailing need to cover more spaces to build houses, squares and buildings has been increasing; However, green spaces, such as urban parks and ecological reserves, have been reduced or abandoned by the corresponding administrations to satisfy the needs of society, with the aim of knowing the importance of the development and conservation of Ecological Reserves or Protected Natural Areas. Looking for an advantage through ecotourism, implementing sustainable marketing strategies, a qualitative study was carried out through the reading of documents from 182 web pages of protected natural areas in Mexico, analyzing characteristics, ecosystems and facilities, deepening the documentary review in the three main Ecological reserves of Querétaro. The main findings are the lack of an adequate strategy for its resources, the low percentage of budget and investment allocated to safeguarding or improving them; making sustainable marketing very important in the management of these reserves.
\end{abstract}

Keywords: Ecological reserves; sustainable marketing; ecotourism.

\title{
INTRODUCCIÓN
}

En la actualidad, el mundo se desarrolla vertiginosamente, buscando espacios disponibles para la creación de infraestructura que haga frente a la demanda poblacional e industrial; sin embargo, poco a poco, los países con mayor crecimiento, han demostrado preocupación e iniciativas para proteger y resguardar zonas naturales que se encuentran dentro de las grandes urbes. Es posible encontrar espacios públicos, conocidos como parques urbanos, y son pequeños pulmones para las ciudades y donde suele haber diferentes instalaciones, que permiten el ocio y descanso de los habitantes de la zona, por otra parte también existen zonas naturales con abundante vegetación, llamadas reservas ecológicas, las cuales proporcionan gran cantidad de oxígeno, por lo que estos parques son parte fundamental del nuevo diseño urbano ya que conlleva potenciales beneficios ambientales, sociales y económicos, por dichos motivos es importante reconocer que la planeación de los parques es esencial para su creación en las ciudades.

El estudio presenta tres apartados el primero muestra el marco teórico donde se presentan los principales autores sobre la mercadotecnia sostenible y turismo sostenible, en el segundo apartado son los resultados de la investigación documental donde se muestran los antecedentes de las Reservas Ecológicas, su importancia y funcionalidad, los requisitos para valorar y clasificar las reservas ecológicas, los modelos de planificación y gestión de espacios protegidos, las Reservas Ecológicas en el mundo y en México, la problemática que manejan para su desarrollo y aplicación del ecoturismo, y el tercero presenta las conclusiones y reflexiones finales

\section{MARCO TEÓRICO}

Como resultado del Desarrollo Sostenible y los ODS, la humanidad ha buscado la manera de revertir el impacto ambiental que fue generado por grandes industrias y empresas por muchos años; la mercadotecnia enfocada al cuidado y protección de un medio ambiente sano y sin afectaciones para generaciones futuras, ha demostrado ser una solución a largo plazo. La sostenibilidad en la mercadotecnia es la mercadotecnia que perdura, pues proporciona soluciones a necesidades actuales y a largo plazo. (Belz, 2013). 
Es por eso que se puede definir, la mercadotecnia sostenible, como un proceso estratégico que involucra la planeación, implementación y control de desarrollo de precio, promoción y distribución de productos, de manera que logre la satisfacción de las necesidades de los clientes, los objetivos de la organización y el proceso sea compatible con los ecosistemas. Para Fuller (1999), la mercadotecnia sostenible representa una evolución de la mercadotecnia, puesto que, combina las perspectivas de la corriente principal con los nuevos conceptos sociales, éticos, ambientales e intergeneracionales (Belz, 2013). Al respecto, Fischer y Espejo (2011), mencionan que el concepto de mercadotecnia verdeecológica se transformó a mercadotecnia verde- sostenible y finalmente, se convierte en mercadotecnia sostenible

Dentro de la mercadotecnia sostenible un punto importante es la necesidad de promover la conservación de las reservas ecológicas y parques sostenibles en las grandes ciudades, donde su crecimiento ha abarcado o consumido gran parte de estos espacios, es fundamental para lograr los objetivos de desarrollo sostenible propuestos por la Organización de las Naciones Unidas (ONU) en el 2015, a pesar de ello, la tarea de conservar áreas verdes no sólo debe enfocarse en la creación de parques urbanos, también es esencial preservar aquellos espacios naturales protegidos, los cuales tienen la importante tarea de ser un pulmón para las ciudades pero que en muchos casos, han sido olvidados, las Reservas Ecológicas o Áreas Naturales Protegidas, son un espacio que no ha sufrido alteraciones significativas por actividades humanas, o que requiere del resguardo o restauración de su ecosistema, a través de normas y programas ecológicos, con el único fin de conservar la biodiversidad a largo plazo. (GeoEnciclopedia. 2016)

Por su parte, la mercadotecnia socioambiental, como parte de la mercadotecnia social, propone la mejora de la biodiversidad y el desarrollo sostenible de la tierra fijando atención preferente al comportamiento humano que puedan afectar los recursos y especies de las reservas ecológicas. (MierTerán, J. 2004)

\section{MÉTODO}

Con el objetivo de conocer la importancia del desarrollo y conservación de las Reservas Ecológicas o Áreas Naturales Protegidas buscando un aprovechamiento a través del ecoturismo implementando estrategias de mercadotecnia sostenible, se realizó un estudio cualitativo a través de la lectura de documentos de áreas naturales protegidas en México, analizando características, ecosistemas e instalaciones profundizando la revisión documental en las tres principales reservas ecológicas de Querétaro. Las bases consultadas fueron la Comisión Nacional de Áreas Naturales Protegidas (CONANP), (Conanp, 2016), Sistema de Monitoreo y Evaluación para la Conservación (SIMEC) (Simec, 2020), Secretaria de Turismo Federal (SECTUR), (Sectur, 2020) y Secretaria de Turismo Estatal, (Sectur 2020), y Grupo Ecológico Sierra Gorda, (2020).

Se llevó a cabo un proceso de recopilación de información de lo general a lo particular, mediante una base de datos de las 182 áreas naturales protegidas en México, para enfocar la investigación en la categoría de parques nacionales, analizando características, ecosistemas e instalaciones similares. De igual manera, se evaluó el tipo de actividades ecoturísticas se realizan dentro de estos espacios protegidos con la finalidad de obtener áreas de oportunidad y de mejora al aplicar un plan estratégico de mercadotecnia sostenible. 


\section{RESULTADOS}

\subsection{Antecedentes de las Reservas Ecológicas}

Se considera que la primera reserva ecológica surgió en Norteamérica en 1872, con la creación del Parque Nacional Yellowstone ubicado en Wyoming, con el único objetivo de proteger íntegramente un espacio natural virgen. Durante la Convención Internacional para la Protección de la Flora y Fauna en su Estado Natural de Londres en 1933, se establecieron los elementos fundamentales del concepto de Parque Nacional, definiéndolo como un área puesta bajo control público para el aprovechamiento y esparcimiento público, para interés científico y de la cual no podrá transferirse ninguna parte salvo por la autoridad competente. (Ambientum. 2015)

Como resultado de los beneficios atribuidos a Yellowstone, el fenómeno por proteger áreas naturales fue creciendo, consiguiendo que en 1950 existieran más de 400 Parques Nacionales en 70 países; finalmente, en 1962 se llevó acabo la Primera Conferencia Mundial de Parques Nacionales y Reservas Equivalentes en Seattle, Estados Unidos; posteriormente este tipo de conferencias se realizan cada 10 años, con el fin de influenciar y detallar las perspectivas sobre la sostenibilidad del medio ambiente a través de las Áreas Naturales Protegidas. (Monroy Ojeda, A. 2019)

En 1972, tomó lugar la Convención para la Protección del Patrimonio Mundial Cultural y Natural, con el objetivo de evaluar e identificar los bienes inestables e irremplazables a nivel mundial, ya que en caso de la pérdida de alguno de ellos sería un daño social de tamaño invaluable, así pues, la Organización de las Naciones Unidas para la Educación, la Ciencia y la Cultura (UNESCO) creó la 'Lista del Patrimonio Mundial' como instrumento sostenible para proporcionar información de los monumentos y sitios de gran riqueza natural y cultural, así como para la transmisión de ese patrimonio a las generaciones futuras. (UNESCO, 2006)

Con el surgimiento del término 'sostenibilidad' y su dimensión ambiental, las reservas ecológicas han tomado fuerza e interés por parte de un público más consciente del impacto que generan los actos humanos e industriales; el correcto resguardo de estas zonas, beneficiará de manera sustentable a las ciudades en crecimiento, ya que la preservación de la biodiversidad de las áreas naturales protegidas ofrecerá, no sólo un espacio con gran belleza escénica, sino que será el vínculo permanente entre los individuos y la naturaleza a través del tiempo.

Por esta razón, la educación ambiental debe de ser una actividad intrínseca en todas las reservas ecológicas, pues los valores naturales y culturales que se encuentran inmersos en estos espacios, contribuyen fundamentalmente al proceso de educar ambientalmente a la sociedad a través de la función recreativa, como derecho de todo ciudadano a disfrutar de un medio ambiente de calidad. (Tolón, A. 2008)

\subsection{Funcionalidad e importancia de las áreas naturales protegidas}

El principal objetivo de las reservas ecológicas, es servir de protección a los ecosistemas que albergan y promover el interés de la sociedad por preservar el medio ambiente, invitando a la población a familiarizarse y conocer la naturaleza que se desarrolla en la zona, así mismo estas reservas ecológicas son base de investigaciones y estudios de carácter científico, con el fin de ampliar los conocimientos técnicos de la flora y fauna de los ecosistemas inmersos en estas zonas. Generalmente, la gestión de 
estas reservas ecológicas es de carácter gubernamental.

Las Áreas Naturales Protegidas se encuentran en todo el mundo, lo que permite un desarrollo sostenible global por medio de actividades económicas como el ecoturismo, sin embargo, la protección de estos espacios conlleva a beneficios ambientales mayores para la sociedad, como para el entorno en el que se encuentra. A continuación se enlistas algunos aspectos que resaltan la importancia de las reservas ecológicas (Blanco, 2016):

- Captura y secuestro de las emisiones de carbono ${ }^{1}$

- Preservación de flora y especies endémicas que ayudan a la conservación de la biodiversidad del planeta.

- Resguardo de paisajes con gran valor natural y cultural, los cuales albergan formaciones únicas o elementos con antigüedad de cientos de años.

- Aumento de la calidad de vida de la población a través de actividades deportivas y de recreación. (Sánchez, J. 2018)

- Protección de cuencas hidrológicas a modo de asegurar la captación, flujo y calidad del agua.

- Impulsar armónicamente la integración de las poblaciones y la naturaleza, a fin de promover el desarrollo sostenible. (Monroy, A. 2019)

- Proteger las áreas que ofrezcan un interés singular desde el punto de vista científico, cultural, educativo, estético, paisajístico y recreativo o turístico.

- Colaborar en programas internacionales de conservación delabiodiversidad. (Blanco, 2016)

La Comisión Nacional de Áreas Naturales Protegidas (CONANP, 2017) declara que:

"Las áreas naturales protegidas son las herramientas más efectivas para conservar los ecosistemas, permitir la adaptación de la biodiversidad, enfrentar los efectos del cambio climático y ampliar los corredores naturales que permiten que las especies se adapten y ajusten sus áreas de distribución, frente a las nuevas condiciones climáticas".

En contraste, también existen amenazas para las Áreas Naturales Protegidas (ANP), éstas son (CONANP, 2017):

- Cambio de uso de suelo.

- Sobreexplotación de recursos naturales.

- Actividades productivas no sustentables.

- Falta de planeación integral del territorio.

- Introducción de especies exóticas invasoras.

- Contaminación.

- Tráfico ilegal de especies.

A pesar de estas amenazas, la CONANP en México, ha desarrollado modelos de desarrollo sostenible que promueve comités de vigilancia, plantaciones forestales y abonos orgánicos, así como la

1 Captura y secuestro del carbono es la extracción y almacenamiento de carbono de la atmósfera en sumideros (océanos, bosques o la tierra) a través de procesos físicos o biológicos, como la fotosíntesis (GreenFacts.org, recuperado de https://www.greenfacts.org/es/glosario/abc/captura- carbono.htm) 
organización comunitaria para el desarrollo de proyectos ecoturísticos o de rescate de la cultura y las tradiciones.

\subsection{Requisitos de valoración y clasificación de los parques ecológicos}

Hace 25 años, la Unión Internacional para la Conservación de la Naturaleza (UICN) desarrolló un sistema preliminar de categorías para la gestión e identificación de las áreas protegidas de acuerdo a sus características, con el fin de generalizar el entendimiento de estas zonas de manera internacional, actualmente este sistema es punto de referencia para definir y clasificar las áreas protegidas.

Dependiendo de sus atributos y objetivos, las reservas ecológicas pueden ser clasificadas en seis grupos. (Como se muestra en la Tabla 1)

Tabla 1. Categorías de gestión de áreas naturales

\begin{tabular}{|c|c|}
\hline Categoría/ Subcategoría & Objetivo \\
\hline $\begin{array}{l}\text { Categoría I. Protección estricta } \\
\text { Ia. Reserva Natural Estricta }\end{array}$ & $\begin{array}{l}\text { Conservar a escala regional, nacional o } \\
\text { global ecosistemas, especies (presencia o } \\
\text { agregaciones) y/o rasgos de geo-diversidad } \\
\text { extraordinarios: atributos conformado } \\
\text { (principalmente o exclusivamente) por fuerzas } \\
\text { no humanas y se degradarían o destruirán si se } \\
\text { viesen sometidos a cualquier impacto humano } \\
\text { significativo. }\end{array}$ \\
\hline Ib. Área Natural silvestre & $\begin{array}{l}\text { Proteger la integridad ecológica a largo } \\
\text { plazo de áreas naturales no perturbadas por } \\
\text { actividades humanas significativas, libres } \\
\text { de infraestructuras modernas y en las que } \\
\text { predominan las fuerzas y procesos naturales, de } \\
\text { forma que las generaciones presentes y futuras } \\
\text { tengan la oportunidad de experimentar dichas } \\
\text { áreas. }\end{array}$ \\
\hline $\begin{array}{l}\text { Categoría II. Conservación y protección del } \\
\text { ecosistema } \\
\text { Parque Nacional }\end{array}$ & $\begin{array}{l}\text { Proteger la biodiversidad natural junto con la } \\
\text { estructura ecológica subyacente y los procesos } \\
\text { ambientales sobre los que se apoya, y promover } \\
\text { la educación y el uso recreativo. }\end{array}$ \\
\hline $\begin{array}{l}\text { Categoría III: Conservación de los rasgos naturales } \\
\text { Monumento natural }\end{array}$ & $\begin{array}{l}\text { Proteger rasgos naturales específicos } \\
\text { sobresalientes y la biodiversidad y los hábitats } \\
\text { asociados a ellos. }\end{array}$ \\
\hline $\begin{array}{l}\text { Categoría IV: Conservación mediante manejo } \\
\text { activo } \\
\text { Área de manejo de hábitats / especies }\end{array}$ & $\begin{array}{l}\text { Mantener, conservar y restaurar especies y } \\
\text { hábitats. }\end{array}$ \\
\hline $\begin{array}{l}\text { Categoría V: Conservación de paisajes terrestres } \\
\text { y marinos y recreación Paisaje terrestre y marino } \\
\text { protegido }\end{array}$ & $\begin{array}{l}\text { Proteger y mantener paisajes terrestres/marinos } \\
\text { importantes y la conservación de la naturaleza } \\
\text { asociada a ellos, así como otros valores creados } \\
\text { por las interacciones con los seres humanos } \\
\text { mediante prácticas de manejo tradicionales. }\end{array}$ \\
\hline
\end{tabular}


Categoría VI: Uso sostenible de los recursos Proteger los ecosistemas naturales y usar los naturales

Área protegida manejada recursos naturales de forma sostenible, cuando la conservación y el uso sostenible puedan beneficiarse mutuamente.

Fuente: Unión Internacional para la Conservación de la Naturaleza (UICN)

Desde esta perspectiva, el turismo en los parques nacionales puede plantearse como un instrumento para la gestión, ordenación y valor territorial. El desarrollo de esta actividad debe fundamentarse en principios de integridad, sostenibilidad y especialización productiva, como el ecoturismo. Es importante resaltar que, los elementos del patrimonio natural y cultural no son únicamente una atracción recreativa o turística, sino que son parte de un ecosistema y componentes de la memoria colectiva de las sociedades que las han preservado. (Anton, 2008)

\subsection{Modelos de planificación y gestión de espacios protegidos}

Blanco y De Esteban (2016) mencionan que habitualmente cada nación dispone de una ley general para espacios protegidos, donde se establecen los procedimientos para determinar las actividades incompatibles y las condiciones para el desarrollo de aquellas actividades compatibles con el medio. Cada área protegida requiere un plan de gestión que describa la administración de los recursos naturales y culturales, la tabla 2 describe los tipos de gestión y planificación de las áreas naturales protegidas.

Tabla 2. Niveles de planificación y gestión de espacios protegidos

\begin{tabular}{|c|c|c|}
\hline Instrumento & Función & Contenidos \\
\hline Plan director o ley estatal & $\begin{array}{l}\text { Establece el modelo de } \\
\text { gestión de una red de espacios } \\
\text { protegidos. }\end{array}$ & $\begin{array}{l}\text { Directrices para planificación, } \\
\text { declaración y elaboración de } \\
\text { planes de gestión. }\end{array}$ \\
\hline $\begin{array}{l}\text { Plan de ordenación de recursos } \\
\text { naturales o ley declaración }\end{array}$ & $\begin{array}{l}\text { Valora recursos protegibles } \\
\text { y propone delimitación del } \\
\text { espacio }\end{array}$ & $\begin{array}{l}\text { Inventario de recursos } \\
\text { naturales, evaluación del valor } \\
\text { de los recursos, propuesta de } \\
\text { declaración, régimen preventivo. }\end{array}$ \\
\hline Plan de gestión & $\begin{array}{l}\text { Marco legal para la gestión } \\
\text { de las actividades, marco } \\
\text { pragmático y presupuestarios }\end{array}$ & $\begin{array}{l}\text { Zonificación, normativa } \\
\text { de actividades, programas, } \\
\text { presupuestos, órganos de gestión. }\end{array}$ \\
\hline Planes sectoriales & $\begin{array}{l}\text { Establece modelos para } \\
\text { actividades compatibles }\end{array}$ & $\begin{array}{l}\text { Actividades de uso público y } \\
\text { turístico, condiciones de uso, } \\
\text { sistemas de seguimiento. }\end{array}$ \\
\hline
\end{tabular}

Fuente: Blanco y De Esteban, (2016) (pág. 42)

De acuerdo a los tipos de planificación, el plan sectorial se ajusta a las actividades de recreación relacionadas al ecoturismo; según los planes sectoriales se pueden clasificar en (Blanco, 2016):

- Conservación; actuaciones de recuperación de especies y ecosistemas.

- Uso público; programas de equipamientos, interpretación del patrimonio.

- Investigación; seguimiento de impacto de actividades, cambio climático, etc.

- Comunicación; procedimientos de participación y comunicación con los actores implicados y con 
la sociedad.

- Desarrollo sostenible; régimen de actividades y herramientas de financiación para el desarrollo local.

- Evaluación y seguimiento; procedimientos e indicadores de resultados de la gestión.

Con respecto al uso público y los espacios protegidos, el ecoturismo se relaciona con el disfrute de los ciudadanos y su esparcimiento, las actividades de uso público en estas zonas tienen lugar aprovechando recursos naturales y culturales preservando los objetivos de conservación por medio de la infraestructura y equipamientos adecuados para los visitantes; el concepto de uso público tiene una serie de funciones básicas relacionada con los visitantes y los espacios protegidos: divulgación, información y orientación, recreación, interpretación del patrimonio, educación ambiental, señalización y servicios turísticos. (Blanco, 2016)

\subsection{Reservas Ecológicas en el mundo}

Conforme a la Lista de Patrimonio Mundial de la UNESCO, actualmente existen 1,121 sitios inscritos, de los cuales 213 son bienes naturales, 869 bienes culturales y 39 mixtos situados en 193 Estados Miembros (UNESCO. 2020); por su parte, la Base de Datos Mundial de Áreas Protegidas (WDPA, por sus siglas en inglés) registra un total de 258,389 áreas naturales protegidas, gran parte de estas reservas ecológicas son áreas terrestres, cubriendo un total de 20,270, $904 \mathrm{~km}^{2}$, lo equivalente al 15 $\%$ del total de la superficie terrestre de la tierra, y en su mayoría están inscritas bajo la categoría de parques nacionales o áreas de conservación. Las áreas marinas protegidas cubren una superficie de 26, 984, $530 \mathrm{~km}^{2}$, que representa el $7.4 \%$ de los océanos mundiales. (Protected Planet. 2020)

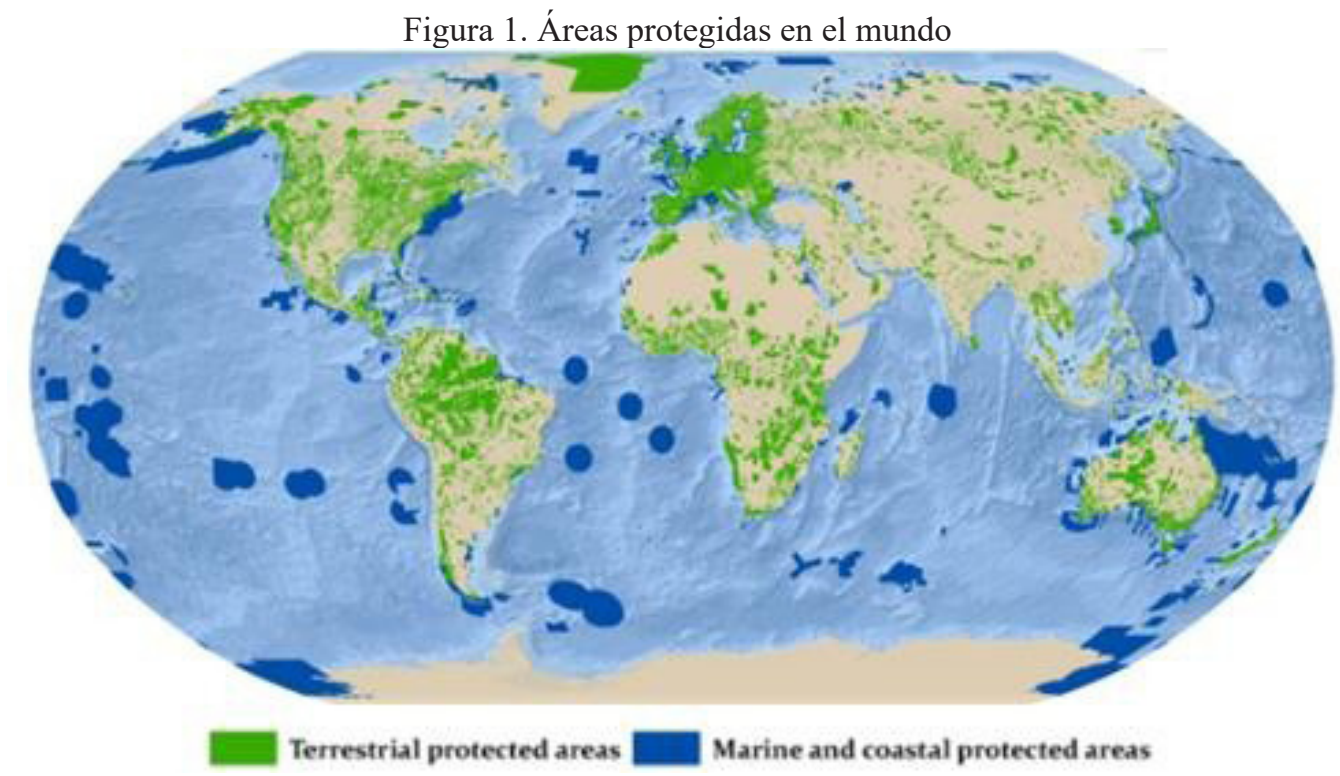

Fuente: PNUAM-WCMC (2019). Áreas protegidas del mundo. Disponible en https://www.protectedplanet.net/c/ monthly-updates/2019/december-2019-update-of-the- wdpa

La mayor parte de territorio protegido se encuentra en América Latina y el Caribe, cubriendo 5 millones de $\mathrm{km}^{2}$, de los cuales Brasil es responsable de 2.47 millones de $\mathrm{km}^{2}$. De manera opuesta, Medio Oriente tiene la tasa más baja de protección de tierras con sólo $119,000 \mathrm{~km}^{2}$, representando menos de un $3 \%$ del total protegido a nivel mundial. (UICN. 2016) 
A pesar del incremento de espacios protegidos en los últimos años, la UICN (2016), declara que sólo el 20 \% de las áreas clave para la biodiversidad se encuentran inscritas en algún programa de protección, más aún, son pocas las áreas que están siendo gestionadas correcta y eficazmente, pues únicamente el $20 \%$ de los países han cumplido con los compromisos de conservación de su áreas protegidas. Actualmente se busca incrementar el espacio protegido con la finalidad de que varias áreas queden conectadas para permitir un mejor flujo de elementos naturales; finalmente, la UICN manifiesta que las áreas protegidas son fundamentales para el desarrollo sostenible a través de actividades económicas como el ecoturismo.

Cada una de estas áreas naturales posee atributos únicos y de gran valor ecológico, los cuales están directamente relacionados con el tipo de clima que exista en el país donde se encuentre, el resguardo y mantenimiento de las reservas ecológicas también está vinculado a la conciencia y comportamiento ecológicos que la sociedad tenga, es de reconocerse que países como Suiza, Francia, Dinamarca, Malta y Suecia sean catalogados como los cinco países con mayor cantidad de acciones ambientales; en contraste, Burundi, Bangladesh, República Democrática del Congo, India, Nepal y Madagascar son los países con menor cuidado del medio ambiente. (Ecoosfera. 2018)

Las Metas de Aichi para la Biodiversidad conforman un conjunto de 20 metas agrupadas en cinco Objetivos Estratégicos que deberán alcanzarse para el 2020; estas metas fueron aprobadas en 2010 durante la $10^{a}$ reunión de la Conferencia de las Partes en el Convenio sobre la Diversidad Biológica, con el fin de detener la pérdida de diversidad biológica, asegurar ecosistemas resilientes con suministro de servicios esenciales y contribuir al bienestar humano, integrándose a las estrategias de los ODS (UICN. 2011); bajo esta perspectiva, la Meta 11 ha sido alcanzada a nivel mundial (protección de zonas terrestres y marinas

\section{RESULTADOS}

Con base a la información documental recabada se encontró que México cuenta con más del $10 \%$ de la diversidad mundial, actualmente, la Comisión Nacional de Áreas Naturales Protegidas (2019) tiene a resguardo federal 182 reservas ecológicas, lo equivalente a 90 millones 839 mil 521 hectáreas; de esta superficie total, 21 millones 886 mil 691 hectáreas (11.14\%) corresponde a ecosistemas terrestres continentales, dulceacuícolas e insulares; y 69 millones 458 mil 748 hectáreas (22.05 \%) a ecosistemas marinos. La superficie de las ANP de México es equivalente a los territorios de Portugal, España e Italia juntos. (CONANP, 2019)

A continuación se muestra en la Tabla 3 la distribución y categorización de las 182 ANP en México, y posteriormente, en la Figura 2 se expone como se encuentran repartidas las áreas naturales en todo el territorio mexicano. 
Tabla 3. Distribución de las superficies de las ANP con base a su categoría de manejo.

\begin{tabular}{|c|c|c|c|c|c|c|c|}
\hline $\begin{array}{c}\text { Categoría de } \\
\text { manejo } \\
\text { (Sistema Nacional) }\end{array}$ & Número & $\begin{array}{l}\text { Superficie } \\
\text { total (ha.) }\end{array}$ & $\begin{array}{l}\text { Porcentaje } \\
\text { del total } \\
\text { protegido } \\
\text { con ANP }\end{array}$ & $\begin{array}{c}\text { Superficie } \\
\text { terrestre y de } \\
\text { aguas } \\
\text { continentales } \\
\text { (ha.) }\end{array}$ & $\begin{array}{c}\text { Porcentaje } \\
\text { del total } \\
\text { terrestre y } \\
\text { aguas } \\
\text { continentales }\end{array}$ & $\begin{array}{c}\text { Superficie } \\
\text { marina (ha.) }\end{array}$ & $\begin{array}{l}\text { Porcentaje } \\
\text { del total } \\
\text { marino }\end{array}$ \\
\hline Reservas de la Biosfera & 44 & $62,952,750.50$ & 69.30 & $9,514,127.91$ & 4.84 & $53,438,622.59$ & 16.97 \\
\hline Parques Nacionales & 67 & $16,220,099.30$ & 17.86 & $673,800.53$ & 0.34 & $15,546,298.77$ & 4.94 \\
\hline Monumentos Naturales & 5 & $16,269.11$ & 0.02 & $16,269.11$ & 0.01 & 0.00 & 0.0 \\
\hline $\begin{array}{l}\text { Áreas de Protección de } \\
\text { Recursos Naturales }\end{array}$ & 8 & $4,503,345.22$ & 4.96 & $4,503,345.23$ & 2.29 & 0.00 & 0.0 \\
\hline $\begin{array}{l}\text { Áreas de Protección de } \\
\text { Flora y Fauna }\end{array}$ & 40 & $6,996,864.17$ & 7.70 & $6,668,602.22$ & 3.39 & $328,261.90$ & 0.10 \\
\hline Santuarios & 18 & $150,193.29$ & 0.17 & $4,628.48$ & 0.0 & $145,564.81$ & 0.05 \\
\hline TOTAL & 182 & $90,839,521.55$ & 100 & $21,380,773.48$ & 10.88 & $69,458,748.07$ & 22.05 \\
\hline
\end{tabular}

Fuente: Comisión Nacional de Áreas Naturales Protegidas, 2018, pág. 39

Figura 2. Distribución de las Áreas Naturales Protegidas clasificadas según su categoría de manejo

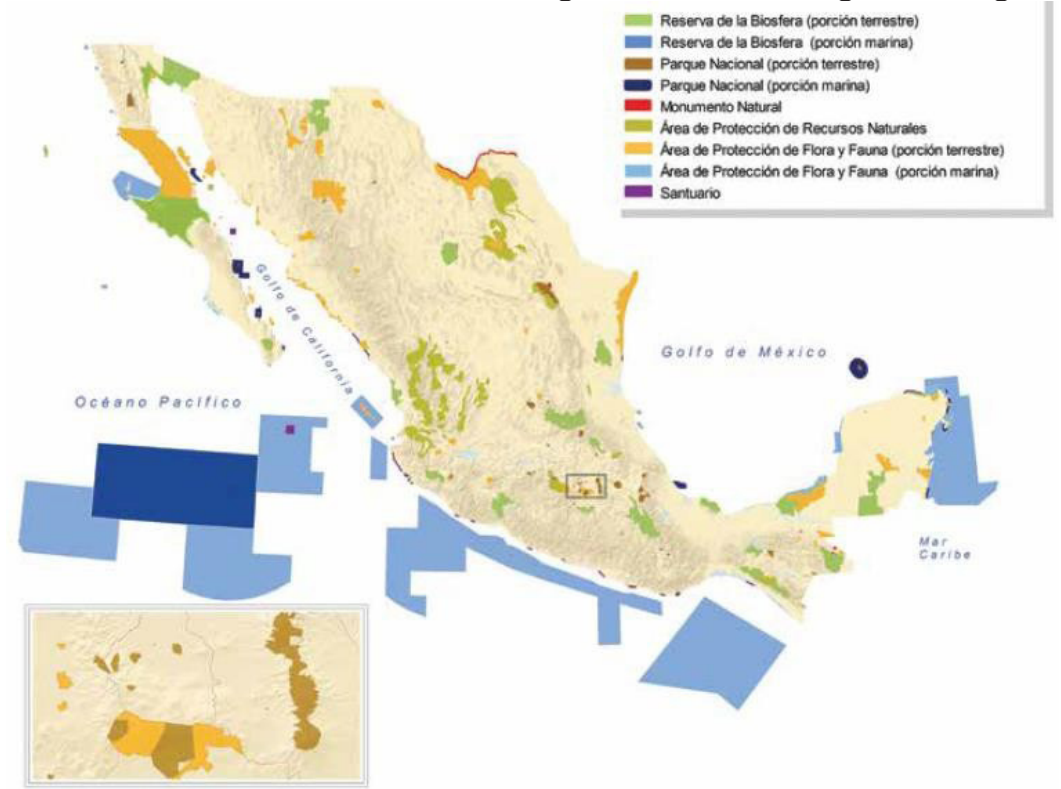

Fuente: Comisión Nacional de Áreas Naturales Protegidas, 2018, pág. 39

De acuerdo a Prezas-Hernández, (2011), para el manejo eficaz de las áreas protegidas, se requiere de un programa administrativo que contenga reglamentaciones ambientales enfocadas a mitigar los daños naturales y representan una herramienta jurídica que delimita la capacidad de carga de los diferentes usos, dicho programa administrativo se le denomina 'programa de manejo'; para México es de suma importancia que las ANP (Áreas Naturales Protegidas), cuenten con un Programa de Manejo, como lo estipula la Ley General del Equilibrio Ecológico y la Protección al Ambiente, (LGEEPA), pues es un instrumento rector de planeación y regulación de actividades, acciones y lineamientos básicos para su correcta operación y manejo. (CONANP, 2018)

Sin embargo, en México, no todas las ANP cuentan con este documento, lo que dificulta la conservación y resguardo de los recursos naturales y al faltar este documento, no existe regulación de las actividades y lineamientos básicos para su manejo, a la fecha se han elaborado 116 Programas de Manejo de un 
total de 182 ANP; concretamente, según los datos actualizados en diciembre 2019 por parte de la CONANP, de los 67 Parques Nacionales decretados solo 39 cuentan con un Programa de Manejo publicado por el SIMEC y de estos, solo 25 fueron inscritos del 2010 a la actualidad. (CONANP, 2019)

Al realizar esta investigación, se hallaron varias limitantes que pueden alterar la correcta elaboración de un plan de mercadotecnia, aplicado a las reservas naturales que deben de ser subsanadas para un adecuado programa de mercadotecnia sostenible, entre las limitantes se encontraron:

a. Poca información de las ANP en México; aunque la SIMEC tiene fichas técnicas de cada área, no existe información suficiente y los datos disponibles en internet no son actualizados o detallados.

b. La capacidad de carga es un estudio costoso y requiere el trabajo conjunto de varias disciplinas, los resultados son dinámicos, pues el medio ambiente cambia constantemente, y esto afecta la valoración final, por lo que la mayoría de los espacios protegidos no cuentan con ello.

c. Gran parte de las reservas ecológicas no tiene publicado su programa de manejo, lo que impide tener un panorama real de la situación y limitaciones de la zona.

d. Poco personal y presupuesto, destinado a las ANP. Al contar con poco presupuesto, es imposible contratar a más personas que puedan resguardar, conservar y promover estos espacios.

\section{CONCLUSIONES}

Uno de los mayores problemas que enfrentan las reservas naturales es el crecimiento del turismo a nivel mundial y nacional, las áreas naturales protegidas se han posicionado como espacios de valor en desarrollo de actividades turísticas, como es el caso del ecoturismo. No obstante, la introducción del turismo genera controversia entre los ambientalistas, al referir que esta actividad en zonas protegidas afecta los ecosistemas del sitio al no contar con estrategias sólidas para controlar la afluencia turística (capacidad de carga) o un programa de manejo adecuado para preservar su valor ambiental es por ello que es necesario recurrir a la mercadotecnia sostenible que permitirá desarrollar estrategias para atraer turismo responsable con el medio ambiente

En México, gran parte de las áreas naturales protegidas no son conocidas entre los turistas nacionales, como resultado del temor por atraer a un turismo en masas, no obstante, es posible ajustar y realizar estrategias mercadológicas para los parques nacionales y reservas ecológicas.

Aunque se reconoce la importancia de las reservas ecológicas, es posible detectar un deterioro gradual en los recursos financieros y humanos, así como la coordinación entre las instituciones gubernamentales, estatales y municipales para la realización de programas protección y recreación, establecimiento de nuevas políticas ambientales y marcos legales que permitan administrar adecuadamente estos espacios. (Tlapa, M. 2020)

\section{REFLEXIONES FINALES}

Por su parte, la mercadotecnia sostenible y socioambiental son enfoques similares pero con prioridades distintas, no obstante, la mercadotecnia socioambiental puede tener un gran alcance en México, pues no hay aún, suficiente bibliografía enfocada a las reservas ecológicas, considerando que México es uno de los países más megadiversos y con gran número de espacios en protección. El área de oportunidad y adopción que la mercadotecnia sostenible puede tener en nuestro país, puede cambiar paulatinamente, la poca cultura ambiental que se tiene hoy en día. 
Las reservas ecológicas son parte fundamental para un desarrollo sostenible, pues sin la dimensión ambiental, la sostenibilidad queda truncada. La mercadotecnia, como método para satisfacernecesidades e influir-de manera indirecta- en el comportamiento de los consumidores, puede ajustarse a la necesidad de conservar el medio ambiente, por medio de acciones y estrategias controladas para motivar el cambio en la conducta de los visitantes. La adopción, paulatina, de la mercadotecnia sostenible ha permitido ampliar el panorama de prevención hacia el medio ambiente, y si se conjunta con el enfoque socioambiental, el resultado podrá traer grandes beneficios para el presente y generaciones futuras

Finalmente, es importante considerar que aunque están bajo protección gubernamental, es factible hallar cierto número de sitios naturales con restricción total para el público en general, derivado de la importancia y fragilidad de los ecosistemas, o bien es sede de alguna investigación científica. En el caso de las Áreas Naturales Protegidas, en las que el acceso es permitido se debe considerar como principal factor de ingreso, un correcto comportamiento pro-ambiental y un conocimiento previo del mantenimiento y resguardo adecuado dentro de las zonas naturales.

\section{REFERENCIAS}

1. Ambientum. 2015. Parques Naturales. Disponible en: https://www.ambientum.com/enciclopedia medioambiental/natura/parques_naturales.asp

2. Anton, S., Blay, J., Salvat, J. 2008. Turismo, actividades recreativas y uso público en los parques naturales: propuesta para la conservación de los valores ambientales y el desarrollo productivo local. Boletín de la Asociación de Geógrafos Españoles, no. 48: 5-38. Disponible en: https://bage. age-geografia.es/ojs/index.php/bage/article/view/712/665

3. Belz, F.M., Peattie, K., y Galí, J. M. 2013. Marketing de sostenibilidad: Una perspectiva global. Barcelona: Profit Editorial.

4. Blanco Portillo, R. y De Esteban Curiel, J. 2016. Ecoturismo. Hacia una gestión sostenible moderna. España: Síntesis.

5. CONANP, 2016, Comisión Nacional de Áreas Naturales Protegidas. 2016, , Disponible en: https:// www.gob.mx/conanp

6. 2017, Comisión Nacional de Áreas Naturales Protegidas). 2017. Las Áreas Naturales Protegidas: respuestas naturales frente al cambio climático. Disponible en: https://www.gob. $\mathrm{mx} /$ conanp/documentos/las-areas-naturales-protegidas-respuestas-naturales-frente-al-cambioclimatico?state $=$ published

7. 2018, Plan Estratégico de Turismo Sustentable para las Áreas Naturales Protegidas. Secretaría de Medio Ambiente y Recursos Naturales. Disponible en: https://www.conanp.gob.mx/documentos/ PNANP20142018.pdf

8. 2019, Áreas naturales protegidas decretadas. Disponible en: http://sig.conanp.gob.mx/website/ pagsig/datos_anp.htm

9. Ecoosfera. 2018,. Cuáles son los países que más y que menos cuidan el medioambiente (ranking). Disponible en: https://ecoosfera.com/2018/02/paises-que-mas-y-que-menos-cuidan-ambientepoliticas-ambientales-verdes-ranking/

10. Fischer, L., y Espejo, J. 2011. Mercadotecnia. Ciudad de México: McGraw- Hill.

11. Fuller, D. A. 1999. Sustainable marketing: managerial-ecological issues. Estados Unidos: SAGE Publications.

12. GeoEnciclopedia. 2016. Áreas Naturales Protegidas del Mundo. Disponible en: https://www. geoenciclopedia.com/areas-naturales-protegidas-del-mundo/

13. Grupo Ecológico Sierra Gorda, 2020. Disponible en: https://sierragorda.net/

14. Mier-Terán, J.J. 2004. Marketing socioambiental: un nuevo paso en el desarrollo del marketing social. International Review on Public and Nonprofit Marketing. DOI: 10.1007/BF02896622

15. Monroy Ojeda, A. 2019. Áreas Naturales Protegidas, un reto mundial. Disponible en: https:// www.inecol.mx/inecol/index.php/es/2013-06-05-10-34-10/17-ciencia-hoy/398-areas-naturales- 
protegidas-un-reto-mundial

16. Prezas-Hernandez, B. 2011. Áreas naturales protegidas en Quintana Roo: un análisis para su conservación. Comisión Nacional para el Conocimiento y Uso de la Biodiversidad (CONABIO).

17. Protected Planet. 2020. Cobertura Global: Capítulo 2. Disponible en: https://livereport. protectedplanet.net/chapter-2

18. PNUAM-WCMC (2019). Áreas protegidas del mundo. Disponible en https://www.protectedplanet. net/c/monthly-updates/2019/december-2019-update-of-the- wdpa

19. Sánchez, J. 2018. ¿Qué es un parque ecológico? Disponible en: https://www.ecologiaverde.com/ que-es-un-parque-ecologico-1212.html

20. Secretaria de Turismo Estatal, 2020. Disponible en: https://www.queretaro.travel/es/

21. Secretaria de Turismo Federal, 2020. Disponible en: https://www.gob.mx/sectur/

22. Simec, 2020. Sistema de Monitoreo y Evaluación para la Conservación. Disponible en: https:// simec.conanp.gob.mx/

23. Tolón, A., Lastra, X. 2008. Los espacios protegidos: concepto, evolución y situación actual en España. Revista electrónica de Medio Ambiente, ISNN-e 1886-3329, no.5. Disponible en: https:// dialnet.unirioja.es/servlet/articulo?codigo $=2712332$

24. Tlapa, M., Bustamante, A., Vargas, S., Cervantes, V., Cruz, G. 2020. Factores del deterioro de las áreas naturales protegidas periurbanas del Valle de Puebla, México. Estudios demográficos y urbanos, vol.35, no. 1. DOI: http://dx.doi.org/10.24201/edu.v35i1.1828

25. UICN. 2011 UICN (Unión Internacional para la Conservación de la Naturaleza). 2011. Integrar las Metas de Biodiversidad de Aichi en los Objetivos de Desarrollo Sostenible. Unión Internacional para la Conservación de la Naturaleza. Disponible en: https://www.iucn.org/sites/dev/files/import/ downloads/aichi_targets_brief_spanish.pdf

26. 2016. El 15\% de las tierras del planeta están protegidas, pero quedan excluidas áreas cruciales para la biodiversidad. Disponible en: https://www.iucn.org/es/news/secretariat/201609/el-15-delas-tierras-del-planeta-est $\% \mathrm{C} 3 \% \mathrm{~A} 1 \mathrm{n}$-protegidas-pero-quedan-excluidas- $\% \mathrm{C} 3 \% \mathrm{~A} 1$ reas-crucialespara-la-biodiversidad\#: : text $=\mathrm{Seg} \% \mathrm{C} 3 \% \mathrm{BAn} \% 20 \mathrm{los} \% 20$ cient $\% \mathrm{C} 3 \% \mathrm{ADficos} \% 20 \mathrm{de} \% 20$ la,con $\% 20$ exclusi $\%$ C3\%B3n $\% 20 \mathrm{de} \% 201 \mathrm{a} \% 20$

27. UNESCO. 2006 (Organización de las Naciones Unidas para la Educación, la Ciencia y la Cultura). Textos básicos de la Convención del Patrimonio Mundial de 1972. Disponible en: https://whc. unesco.org/archive/convention-es.pdf

28. 2020. Lista del Patrimonio Mundial. Disponible en: http://whc.unesco.org/en/list/ 